\title{
Spectral analysis of Floating Car Data
}

\author{
F. Gössel, E. Michler, and B. Wrase \\ Dresden University of Technology, Fakultät für Verkehrswissenschaften "Friedrich List", D-01062 Dresden
}

\begin{abstract}
Floating Car Data (FCD) are one important data source in traffic telematic systems. The original variable in these systems is the vehicle velocity. The paper analyses the measured value "vehicle velocity" by methods of information technology. Consequences for processing, transmission and storage of FCD under condition of limited resources are discussed.

Starting point of the investigation is the analysis of spectral characteristics of velocity-time-profiles. The spectra are determined by the Discrete Fourier Transform (DFT) from measurement data and simulation data of a microscopic traffic model. One essential property of velocity-time-profiles is their low-pass characteristic. The resulting cut-off-frequency is comparatively small and depends on the traffic scenario. Conclusions concerning the necessary sample rate in FCD systems and the processing of raw data are discussed. Finally the transinformation of velocity-time-profiles is analysed. This results in similar values for an optimal sample rate of FCD systems under condition of limited transmission capacity.
\end{abstract}

\section{Introduction (Michler, 2001; Michler et al., 1999)}

The generation of actual and reliable traffic data can be realised only by fusion of systems with different data sources. In this context Floating Car Data (FCD) Systems are becoming more and more important. For the assessment of the actual traffic state by means of floating car data the measured vehicle velocity is the original variable. The velocity data have to be transmitted to a traffic information centre (TIC). Usually data are transmitted as aggregated values for spatial resp. temporal intervals (segments). As the transmission, processing and storage capacity is limited investigations in properties of velocity-time-profiles are of interest.

Correspondence to: F. Gössel

(goessel@vini.vkw.tu-dresden.de)

\section{Traffic data generation by means of Floating Car} Data (FCD) technology

FCD is a method to generate traffic data. Traffic can be defined as the entirety of motion-lines (trajectories) of all vehicles in an observation area (Leutzbach, 1988). These motion-lines may be visualised in a time-distance diagram (see Fig. 1a). The slope of the motion-lines corresponds to the instantaneous velocity (for easier identification of absolute values they are also coded by colour), traffic density and traffic flow can be extracted simply by counting the number of vehicles per distance resp. time (Schnabel, Lohse 1997). In conventional traffic data generation fixed infrastructure (e.g. loops, cameras) is used. This results in local data (instantaneous velocity, traffic flow). The direct measurement of mean speed resp. travel time per segment is not possible. FCD uses some specially equipped probe cars floating with the traffic current as data sources (Gössel et al., 2001). It is a random sample method (Michler, 2000). Travel time and mean speed of probe cars can be measured directly. The limited transmission capacity and the distribution of resources between TIC and probe cars requires transmission of aggregated values at certain times (see Fig. 1b).

\section{Spectral analysis of velocity-time-profiles}

Generally the study of a signal in the frequency domain may result in a better understanding of certain properties. The following analysis is based on data derived from extensive field trials on motorways in Germany and in the city of Dresden. For some special purposes also data of a microscopic traffic simulation model are used. Both the analysis of velocitytime-profiles and velocity-position-profiles is possible. Theoretical these profiles can be obtained from the time-distance diagram by projection of the vehicle velocity on one of the two axis. Practically FCD velocity data are available as velocity-time-data, to obtain velocity-position-data a conversion procedure (resulting in a much lower precision) is re- 


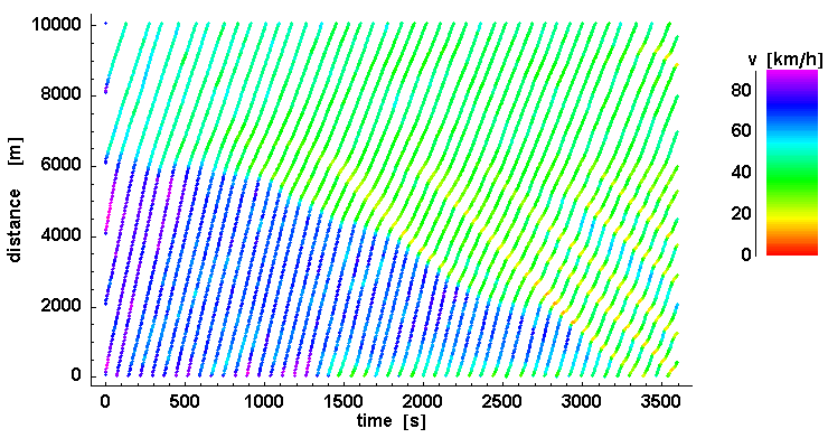

Fig. 1a. Example of a time-distance diagram derived from data of a microscopic traffic simulation.

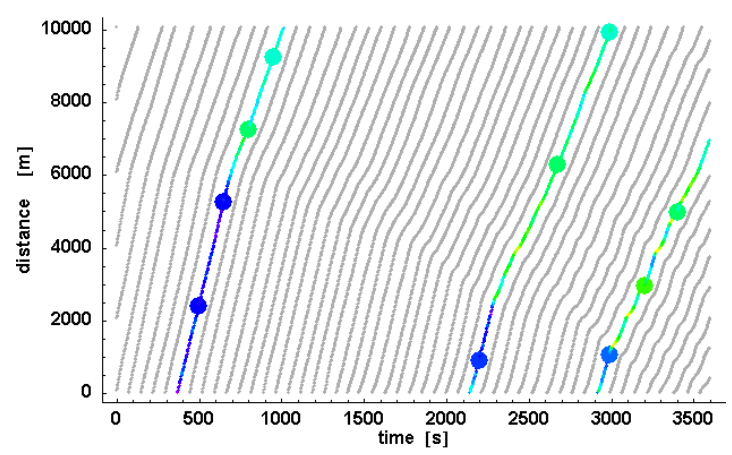

Fig. 1b. Illustration of the FCD method. Trajectories of Probe cars are coloured, Points indicate data transmission.

quired. As velocity-time-data emphasize regions with low speed they are more interesting for practical investigations. In the following therefore only velocity-time-profiles are discussed but results can simply be carried over to velocityposition-profiles. Spectra will be calculated using a discrete Fourier transform as only samples from the velocity-timeprofile are available.

\subsection{Theoretical basics for the use of the DFT (Discrete Fourier Transform)}

To avoid aliasing effects it is necessary to satisfy the sampling theorem. This requires a band limited signal and a sample frequency at least double the highest frequency in the signal. The vehicle velocity is a band limited signal because of the limited acceleration and breaking ability of real vehicles. Velocity data are usually available in the GPS typical sampling distance of one second. Theoretical investigations in hypothetical extreme velocity profiles in a driving dynamics view show a cut-off-frequency below the highest acceptable frequency of $0.5 \mathrm{~Hz}$. A second restriction results from the relation between spatial resolution and frequency resolution. Traffic data are location-based data. For a given sampling frequency a finer frequency resolution means a coarser spatial resolution and vice versa. So a trade-off between frequency and spatial resolution is necessary.
Table 1. Cut-off-frequency for different traffic situations, power criteria $90 \%$

\begin{tabular}{ll}
\hline Scenario & Cut-off-frequency $[\mathrm{Hz}]$ \\
\hline City & 0,04 \\
Highway & 0,01 \\
Rural & 0,02 \\
\hline
\end{tabular}

\subsection{Low-pass property of velocity-time-profiles}

As stated before velocity-time-profiles are band-limited signals for physical reasons. Empirical investigations show a concentration of signal power at very low frequencies $(0 \ldots 0.01 \mathrm{~Hz}$, see Fig. 2).These very low frequencies result from the wish of the driver to keep a certain velocity. The signal power at higher frequencies is not vanishing completely. This is mainly a result of the leck effect and may be reduced by application of a window-function.

For the quantitative determination of the low-pass cut-offfrequency (in analogy to similar problems e.g. frequency modulation) a power criteria is applied: The signal power in the low-pass signal should exceed $90 \%$ (95\%) of the overall signal power (see Fig. 3). Table 1 shows cut-off-frequencies for different traffic situations derived from empirical investigations in real traffic data.

3.3 Consequences of low-pass property for the dimensioning of traffic monitoring systems

Traffic monitoring using the FCD-technology can be based on two access schemes. If the actual velocity (or better the mean velocity of an interval) is polled an optimal polling rate should be determined. For systems using a random access scheme to transmit velocity profiles (of a certain interval at some points or actions of interest) the optimal sampling rate of the velocity profile is of interest. In both cases the necessary sample rate can be derived from the cut-offfrequency. This allows an efficient use of transmission bandwidth and memory. Example: For highway traffic with a cut-off-frequency of $0,01 \mathrm{~Hz}$ a sampling rate of $0,02 \mathrm{~Hz}$ (one sample every $50 \mathrm{~s}$ ) is sufficient.

Another application for the low-pass property is the filtering of GPS raw data. These data usually contain frequent errors due to shadowing. The resulting jumps can be eliminated by low-pass filtering or even simple calculation of a moving average. In the latter case the interval length can be derived from cut-off-frequency (see Fig. 4, the cut-offfrequency not necessarily has to coincide with the first zeropoint, it is also possible to use a certain attenuation criteria).

3.4 Relation between traffic state and spectral characteristics of velocity-time-profiles

The empirical investigations in the relation between traffic state and spectral characteristics of velocity-time- profiles are primarily based on data of a microscopic traffic simu- 

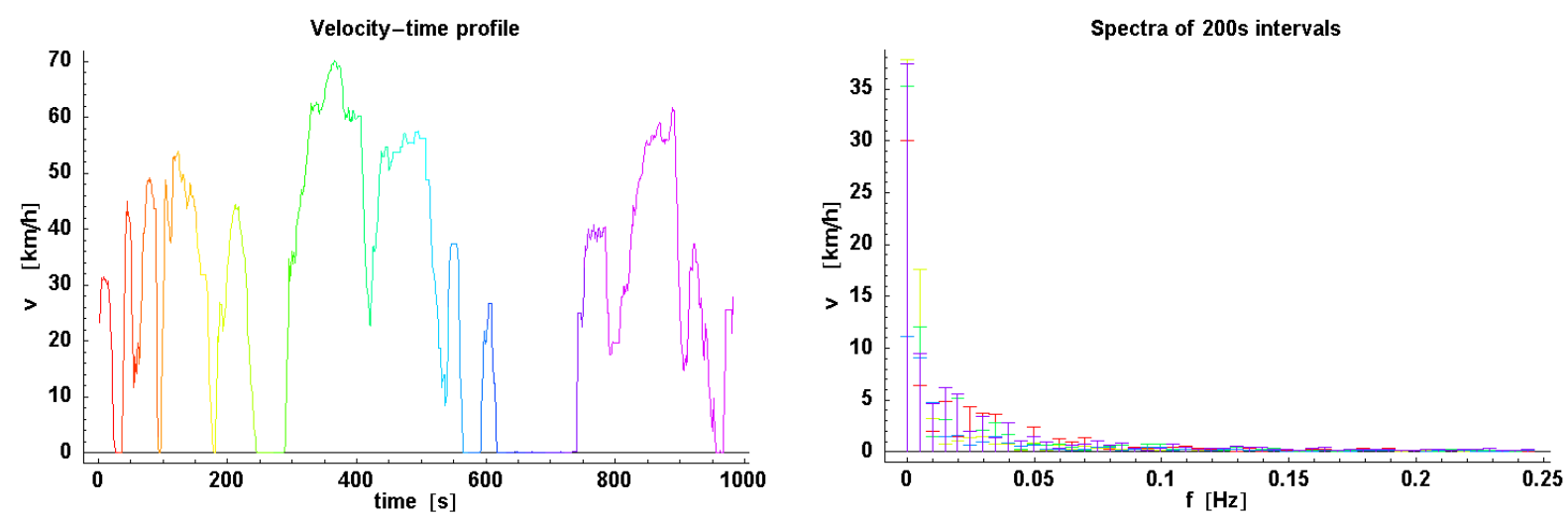

Fig. 2. Velocity-time-profile and corresponding spectra, segments (200s) indicated by colour.

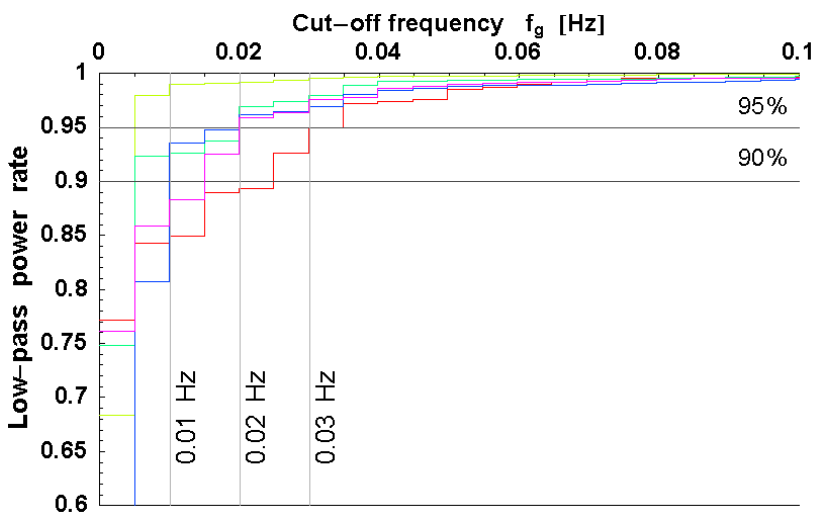

Fig. 3. Power rate of low-pass signal depending from cut-offfrequency, colours corresponding to Fig. 2.

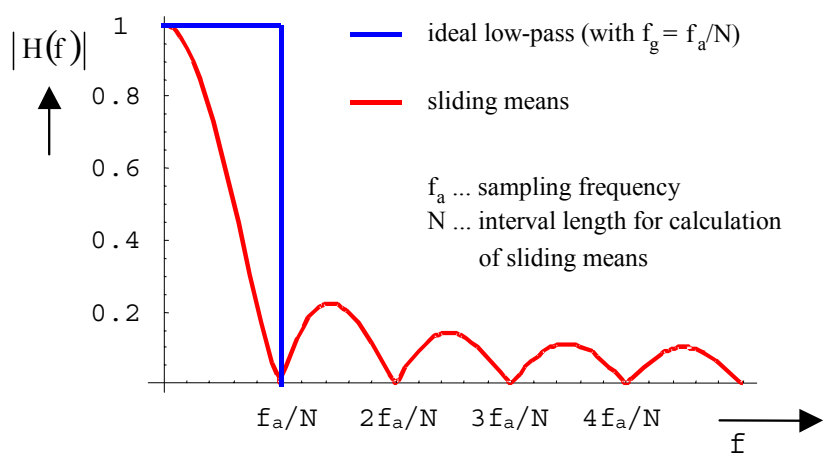

Fig. 4. Frequency response of ideal low-pass filtering and calculation of sliding means.

lation (see Fig. 1). Therefore the simulated space-time area is divided in segments of $2 \mathrm{~km}$ length and $5 \mathrm{~min}$ duration (see Fig. 5). Every segment is assigned to a certain traffic state (free traffic, dense traffic, stop-and-go traffic, transition between these states).

Figure 6 shows spectra of segments with free traffic, stopand-go traffic and transition between free and stop-and-go traffic. Spectra of free traffic and dense traffic show no signif-

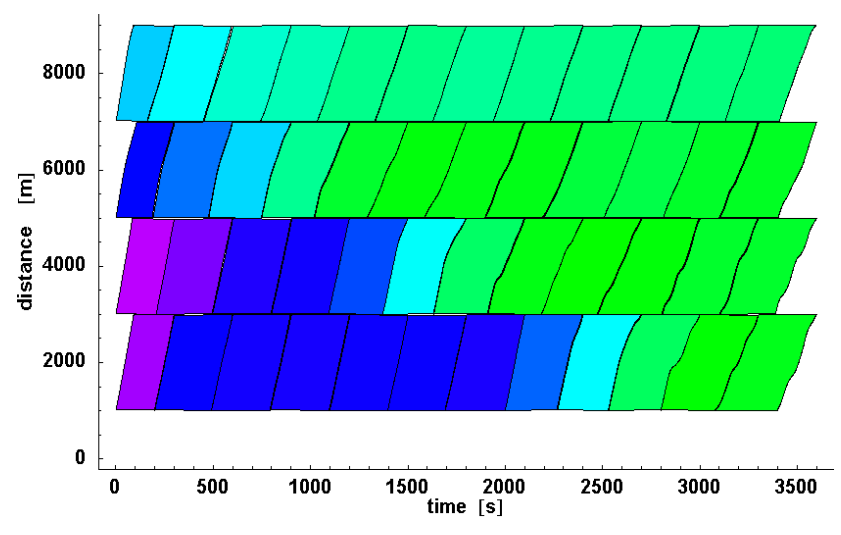

Fig. 5. Segmentation of observation area, colours corresponding to mean speed in segment, legend see Fig. 1.

icant qualitative difference. The power is nearly completely (typical 98...99\%) concentrated on the constant component (mean value), high frequency components are inconsiderable. Spectra of free traffic and dense traffic differ only in the absolute value of the constant component (mean speed). Segments with stop-and-go traffic result in significant power rates at frequencies between $0,01 \mathrm{~Hz}$ and $0,02 \mathrm{~Hz}$. The constant component is reduced on about $80 \%$ of the overall power. An interesting behaviour show segments with transitions between two states. There spectra are basically decreasing with increasing frequency, the constant component contains about $90 \%$ of the overall power. For easier estimation of power relations also the power rates (see Fig. 3) are given. The different colours correspond to different vehicles passing the segment.

\section{Analysis of transinformation in velocity-time-profiles (Outlook)}

Velocity-time-profiles also can be analysed in an information entropical context. Therefore velocity-time-profiles are considered as a sequence of symbols (Ebeling et al., 1998). The alphabet consists of the possible velocity values with a cer- 

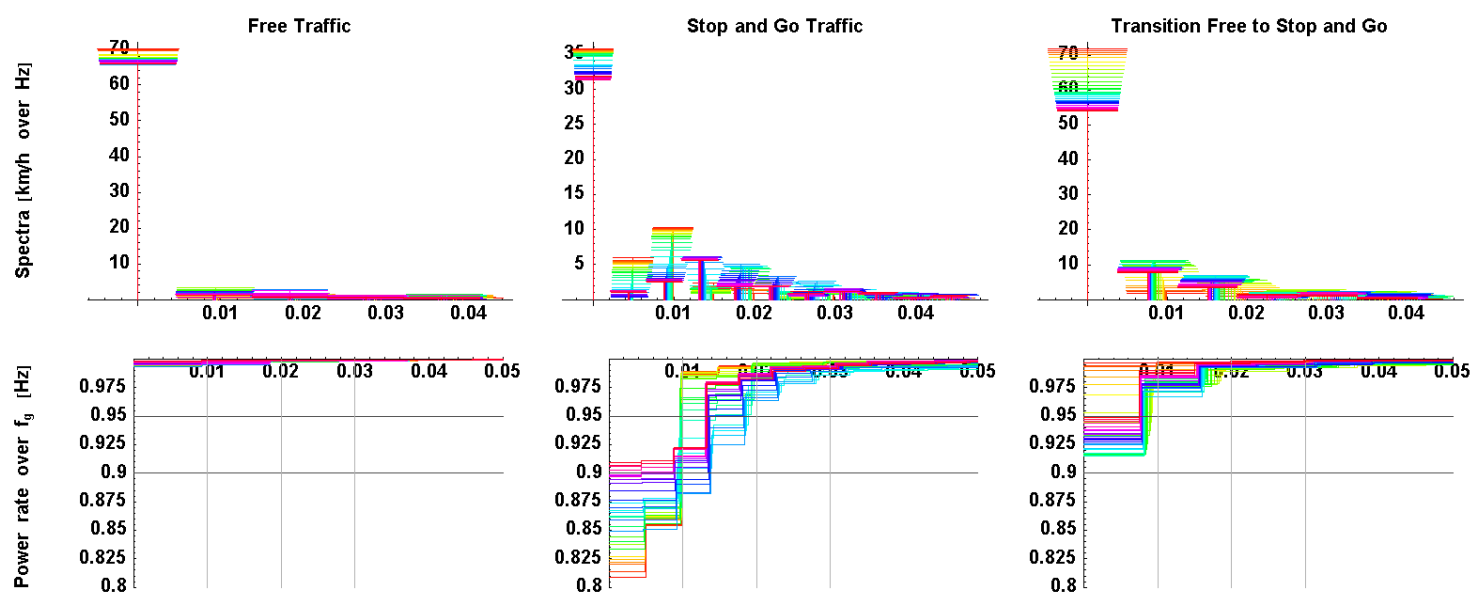

Fig. 6. Spectra and power rates for segments with typical traffic states.

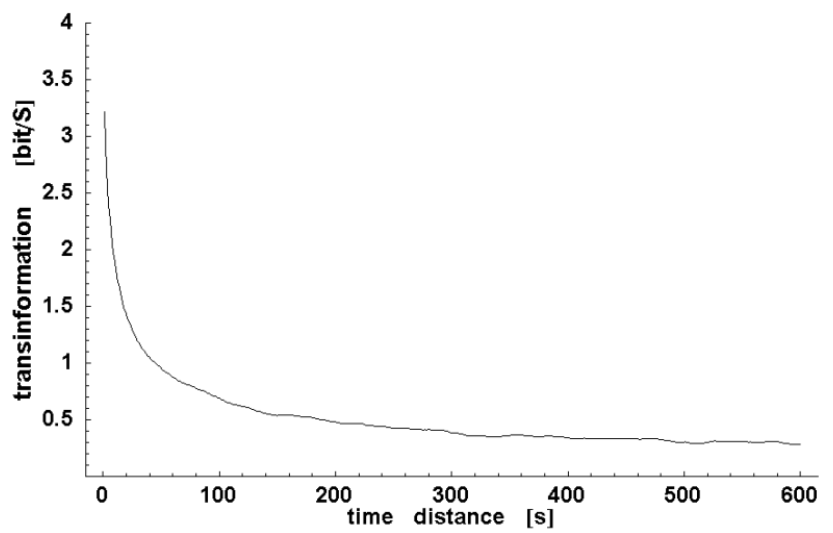

Fig. 7. Transinformation in velocity-time-profiles (data from a journey Munich-Dresden, approx. 20000 values, quantisation $10 \mathrm{~km} / \mathrm{h})$.

tain resolution depending on the available amount of data. To analyse the dynamic of entropy here only the transinformation between letters (velocity values) in distance $n$ (temporal distance in seconds) is considered (see Fig. 7).

A high value of the transinformation shows a statistical dependence, small values are indicating randomness. For small values of $n(1 \ldots 10 \mathrm{~s})$ velocity values show a strong statistical dependence. The reasons are the same as for the low-pass property of velocity-time-profiles: limited acceleration and braking ability of vehicles and the wish of the driver to keep a certain speed level. With raising distance $n$ the transinformation is decreasing up to a nearly constant value (different from zero). This indicates a Markov property of the underlying process.

The characteristic of the transinformation also can be used to determine an optimal sampling distance. Under conditions of a limited transmission capacity every sample should contain an optimum of information. So the transition between the strong statistical dependence meaning a small amount of information and the nearly constant value of the transin- formation (nearly random distribution of values in a certain range resulting in a high information content) becomes interesting as an optimal sampling interval. Empirical investigations show an optimal value between $50 \mathrm{~s}$ and $100 \mathrm{~s}$.

\section{Summary}

Velocity-time-profiles show a low-pass characteristic. The cut-off-frequency depends on the traffic situation and has very small values between 0,01 and $0,04 \mathrm{~Hz}$. This can be used to determine optimal sampling intervals for FCD systems. Another application is the filtering of raw data. Spectra also can be used to determine traffic states from velocitytime-profiles. This becomes interesting especially for automated detection of certain traffic states from velocity data. Finally investigations in information entropy show similar results for optimal sampling rates as the spectral analysis.

Acknowledgements. The authors thank Dr. M. Treiber for the provision of data from a microscopic traffic simulation model.

\section{References}

Ebeling, W., Freund, J., and Schweitzer, F.: Komplexe Strukturen: Entropie und Information, Teubner Stuttgart Leipzig, 1998.

Gössel, F., Wrase, B., and Michler, E.: Neue Ansätze zur fahrzeuggenerierten Verkehrsdatengewinnung in urbanen Regionen: Processing, Analyse und Qualifizierung von Floating-CarDaten, Proceedings, 18. Verkehrswissenschaftliche Tage, TU Dresden, 2001.

Leutzbach, W.: Introduction to the Theory of Traffic Flow, Springer-Verlag Berlin Heidelberg, 1988.

Michler, E., Wrase, B., and Gössel, F.: Fahrzeuggenerierte Verkehrsdatengewinnung mit LEO-FCD-Systemen, Kleinheubacher Berichte 42, 378-387, 1999.

Michler, E.: Verkehrsmonitoring mit fahrzeuggenerierter Verkehrsdatengewinnung. Wissenschaftliche Zeitschrift der Technischen Universität Dresden, 49, Heft 3, 72-76, 2000.

Michler, E.: Verkehrsmonitoring/Verkehrsdatengewinnung, Kleinheubacher Berichte 44, 296-305, 2001.

Schnabel, W. and Lohse, D.: Grundlagen der Straßenverkehrstechnik und der Verkehrsplanung, Berlin: Verlag für Bauwesen, 1997. 